\title{
Educação Mediada por Tecnologia em Disfagia Orofaríngea: Proposta de Ensino na Graduação
}

\section{Technology-Mediated Education in Oropharyngeal Dysphagia: Undergraduate Teaching Proposal}

\author{
Brenda Catalani*, Gabriele Ramos de Luccas, Giédre Berretin-Felix \\ Faculdade de Odontologia de Bauru da Universidade de São Paulo \\ * Autora para correspondência: brenda.catalani@usp.br
}

RESUMO

O termo disfagia refere-se a um sintoma diante de alguma dificuldade no processo de deglutição, causada por disfunção esofágica ou orofaríngea, podendo ocasionar insuficiência nutricional e de hidratação. Sendo assim, é importante a atuação fonoaudiológica; porém, para a realização eficiente do tratamento de indivíduos disfágicos, é necessária formação específica. Com o aumento do uso de tecnologias nas áreas de telecomunicações, tornou-se possível o ensino transmitido por meio de tecnologias de informação e comunicação: a Educação a Distância. O objetivo deste estudo foi verificar a efetividade do uso do ensino a distância, associado ao presencial, no processo de aprendizagem de disfagia dos estudantes de graduação em Fonoaudiologia da Faculdade de Odontologia de Bauru - USP. Assim, participaram desta pesquisa estudantes que tiveram contato com a disciplina apenas de forma presencial, e outros que, além do ensino presencial, tiveram acesso à Plataforma Moodle. Todos os estudantes responderam um questionário com perguntas sobre o conteúdo abordado, e foi aplicado um protocolo de avaliação de habilidades e competências. Além disso, opinaram sobre o material educacional on-line. Na análise dos resultados nos questionários houve diferença estatisticamente significante entre notas pré e pós-disciplina apenas para os estudantes do grupo de ensino híbrido. Quanto ao protocolo de habilidades e competências, as notas atribuídas em cada aspecto foram semelhantes para estudantes de ambos os grupos. No que se refere à percepção dos estudantes quanto ao material on-line, todos referiram que esse ajudou em sua formação. Com isso, conclui-se que o ensino foi efetivo para essa população.

Palavras-Chave: Transtornos de Deglutição; Educação a Distância; Telemedicina.

\begin{abstract}
The term dysphagia is defined as a symptom referring to difficulty during the swallowing process, caused by esophageal or oropharyngeal dysfunction, which may induce nutritional and hydration insufficiency. Thus, the speech therapy proceeding is important, but a specific training is necessary for the efficient performance of the treatment of dysphagic individuals. With the increasing development of technology in the telecommunication area, teaching transmitted by information and communication technologies has become possible. The aim of this study is to verify the effectiveness of distance learning, associated to presential class, of the speech therapy students learning process of the Bauru School of Dentistry - USP. The participants in this research were students who had the discipline in person and others who had access to activities and contents in the Moodle Platform in addition to face-to-face teaching. All students answered a questionnaire before and after the course, with objective questions about the content addressed and a skills and competences evaluation protocol. In addition, they answered questions about online educational material. In the questionnaires results analysis there was a statistically significant difference between pre and post-discipline grades only for the hybrid teaching group students. Regarding the skills and competences protocol, the marks attributed in the end of the discipline in each aspect were similar for students of both groups. Regarding the students' perception about the online material, everyone said that it helped or greatly helped in their professional formation. With this, the authors conclude that this new type of teaching was effective for this population.
\end{abstract}

Keywords: Deglutition Disorders; Distance Education; Telemedicine. 


\section{Introdução}

A deglutição, função vital aos seres humanos, é caracterizada como um processo altamente complexo, dinâmico e importante para a nutrição do organismo (VALE-PRODOMO, ANGELIS \& BARROS, 2010, pp. 61-67). O termo disfagia é definido como um sintoma referente a alguma dificuldade ou desconforto durante qualquer fase do processo de deglutição, podendo ser relacionada a uma disfunção orofaríngea ou esofágica (CLAVÉ et al., 2004, pp. 119-131) e ter causa neurogênica (MILES \& ALLEN, 2015, pp. 4339), mecânica (GOVENDER et al., 2017, p. 43), psicogênica (BAIJENS, KOETSENRUIJTER \& PILZ, 2013, p. 260-270) e relacionada ao envelhecimento (presbifagia) (WIRTH et al., 2016, pp. 189-208). Caso não sejam identificadas e tratadas, as disfagias podem ocasionar pneumonias aspirativas (VALE-PRODOMO, CARRARA DE ANGELIS \& BARROS, 2010, pp. 61-67) e quadros de insuficiência nutricional e de hidratação (ABDULMASSIH et al., 2009, pp. 55-62).

Diante dessas consequências, a intervenção precoce no quadro das disfagias é fundamental, sendo que a avaliação e tratamento são realizados de maneira multidisciplinar envolvendo a atuação de médicos, fonoaudiólogos, nutricionistas, fisioterapeutas, dentistas, entre outros. Em relação à atuação fonoaudiológica, a avaliação clínica da deglutição é composta por: levantamento da queixa e história clínica e aplicação de questionários para verificar sintomas (GONÇALVES, REIMALLI \& BEHLAU, 2013, pp. 601-4); observação das estruturas e aspectos morfológicos que atuam de maneira indireta na deglutição, tais como sensibilidade, mobilidade e tonicidade orofacial; mastigação e avaliação direta da deglutição de alimentos em diversas consistências (líquido, pastoso e sólido). Durante a avaliação direta, são observados os seguintes aspectos durante a deglutição: vedamento labial, escape oral anterior, movimento da língua, permanência do alimento na boca, nasorregurgitação, elevação laríngea, ausculta cervical, alteração respiratória, vocal ou na coloração facial, engasgos, tosse ou pigarro, resíduos alimentares, número de deglutições e oximetria (PADOVANI et al., 2007, pp. 199-205).

Os resultados da avaliação clínica determinam a necessidade da análise instrumental da deglutição, sendo a nasolaringoscopia e a videofluoroscopia os exames mais utilizados para tal finalidade (PADOVANI et al., 2013, pp. 1-7), já que permitem a observação direta de diferentes episódios que podem ocorrer nas diferentes fases da deglutição. Além disso, questionários que analisam a qualidade de vida (PORTAS; GUEDES, 2012, pp. 169-92) e o estado nutricional (MACHADO, COELHO \& VERAS, 2015, p. 132) destes pacientes podem ser aplicados como forma de compreender as consequências desse distúrbio de maneira mais ampla.

O tratamento fonoaudiológico da disfagia orofaríngea é composto por exercícios miofuncionais orofaciais, vocais e respiratórios e manobras protetoras e/ou facilitadoras da deglutição (SPEYER et al., 2010, pp. 40-65), sendo que outros métodos terapêuticos podem ser utilizados para complementar as estratégias clínicas e potencializar os resultados, de acordo com a etiologia de cada disfagia, tais como o uso de biofeedback eletromiográfico (CRARY et al., 2004, pp. 160-4) e a eletroestimulação (LANGMORE et al., 2016, pp. 1221-31; PARK et al., 2016, pp. 426-34). A literatura contempla estudos sobre a atuação fonoaudiológica em casos de disfagia orofaríngea neurogênica (MILES \& ALLEN, 2015, pp. 433-9), mecânica (PERRY et al., 2016) e presbifagia (SANTORO et al., 2011, pp. 61-6).

$\mathrm{O}$ profissional recém-graduado em Fonoaudiologia possui habilitação para atuar em todas as especialidades fonoaudiológicas, incluindo a área de disfagia orofaríngea (CONSELHO FEDERAL DE FONOAUDIOLOGIA, 2016). Porém, considerando-se que essa área de atuação requer o domínio de conhecimentos teórico-práticos específicos para a condução eficiente, segura e adequada de casos de disfagia, é necessário que o fonoaudiólogo tenha acesso a esses conteúdos ainda na graduação.

$\mathrm{O}$ curso de graduação em Fonoaudiologia da Faculdade de Odontologia de Bauru da 
Universidade de São Paulo oferece a disciplina clínica de disfagia orofaríngea semestralmente para estudantes do quarto ano, como disciplina optativa eletiva, com carga horária de 60 horas e que tem por objetivo desenvolver habilidades voltadas ao diagnóstico e tratamento de quadros de disfagia orofaríngea em adultos e idosos, além de estabelecer o papel do fonoaudiólogo na equipe interdisciplinar. Após a conclusão da disciplina, os estudantes relatam com frequência sobre a necessidade de criação de uma plataforma on-line para disponibilizar conteúdos e atividades com o objetivo de aprofundar o conhecimento adquirido, uma vez que a carga horária da disciplina dentro do curso de graduação é pequena.

Com o aumento do uso da internet e aperfeiçoamento da tecnologia em sua totalidade, as barreiras físicas das salas de aula convencionais estão sendo superadas para desencadear um novo processo de aprendizagem em que o ensino é transmitido por meio de tecnologias de informação e comunicação, caracterizando-se como Educação a Distância (EaD) (WEN, 2008, p. 9). Esta modalidade de ensino se mostra útil diante da necessidade de flexibilizar e potencializar os horários e tempo de estudo dos estudantes, aprimorar as habilidades aprendidas por meio de atividades complementares e intermediar a troca de informações entre estudantes, professores e discentes de pós-graduação (PICCOLI, AHMAD \& IVES, 2001, pp. 401-426).

Tendo em vista que as publicações sobre $\mathrm{EaD}$ em disfagia orofaríngea são escassas e voltadas para a formação de profissionais fonoaudiólogos (CATALANI, LUCCAS \& BERRETIN-FELIX, 2016, pp. 638-648), torna-se necessário o desenvolvimento de novas pesquisas que abordem o ensino na graduação, para atender às demandas durante a fase de formação profissional, suprir as lacunas de conhecimento e comprovar a eficiência dessa modalidade de ensino.

Portanto, o objetivo do presente estudo foi verificar a efetividade do uso do ensino a distância, associado ao ensino presencial, para o processo de aprendizagem dos estudantes de graduação de
Fonoaudiologia na área de disfagia orofaríngea.

\section{Métodos}

O trabalho foi desenvolvido na Faculdade de Odontologia de Bauru da Universidade de São Paulo - FOB/USP após a aprovação do Comitê de Ética em Pesquisa dessa instituição, sob o protocolo número 59795416.0.0000.5417.

Os estudantes matriculados na "Disciplina Clínica de Disfagia Orofaríngea - estágio supervisionado" do segundo semestre de 2016 e primeiro semestre de 2017 foram convidados a participar da pesquisa, sendo que oito estudantes participaram no segundo semestre de 2016 (grupo 1) e sete estudantes no primeiro semestre de 2017 (grupo 2), mediante assinatura do Termo de Consentimento Livre e Esclarecido.

Para verificar a efetividade do ensino a distância, associado ao presencial, o grupo 1 realizou a disciplina apenas de forma presencial, enquanto o grupo 2 obteve atividades presenciais associadas com o ensino a distância (ensino híbrido), sendo que ambas as turmas tiveram a mesma carga horária e conteúdo dentro da disciplina.

\section{Ensino Presencial}

A modalidade de ensino presencial, realizada por ambos os grupos de forma obrigatória, foi composta por:

- Aulas expositivas ministradas pela docente responsável, pós-graduandos e profissionais convidados sobre os temas: indicadores do desconforto do trato vocal, do estado nutricional, do estado mental e da qualidade de vida; inter-relação entre a fonoaudiologia, medicina, psicologia, fisioterapia e nutrição no diagnóstico e planejamento terapêutico; diagnóstico clínico e instrumental das disfagias orofaríngeas em adultos e idosos; técnicas de reabilitação fonoaudiológica nas disfagias orofaríngeas em adultos e idosos; tratamento interdisciplinar das disfagias orofaríngeas; orientação aos pacientes e/ou cuidadores;

- Observação de atendimentos realizados pela docente responsável e por discentes de pós-graduação. 
- Atendimento individual de pacientes com disfagia orofaríngea na Clínica de Fonoaudiologia da instituição.

- Apresentação de artigos científicos que abordaram o tema de disfagia orofaríngea relacionado à doença de base apresentada pelo paciente em formato de seminário.

- Entrega de relatório de planejamento terapêutico do paciente e uma planilha terapêutica com a descrição dos objetivos e estratégias abordados a cada sessão, além de relatório final após execução do planejamento terapêutico.

- Prova dissertativa realizada ao final da disciplina, com o objetivo de avaliar o conhecimento teórico e o desenvolvimento da correlação da prática clínica baseada em evidências científicas.

Todas as atividades propostas durante o estágio foram conduzidas e orientadas pela docente responsável, estudantes de pós-graduação e demais docentes e funcionários de modo colaborativo por meio de supervisões clínicas, discussões e estudo dirigido dos casos atendidos.

\section{Ensino Híbrido}

Os estudantes do grupo 2 participaram das mesmas atividades descritas anteriormente no ensino presencial, porém além disso tiveram acesso ao conteúdo de forma on-line na Plataforma Moodle (Modular Object-Oriented Dynamic Learning Environment), um ambiente virtual de aprendizagem que tem como objetivo complementar o processo de ensino, oferecendo gerenciamento de conteúdo acadêmico e recursos de interação e comunicação entre docentes e discentes, com a possibilidade de o professor customizar a plataforma de acordo com suas necessidades e preferências e acompanhar e avaliar as atividades realizadas pelos estudantes por meio da análise de acessos e respostas nas atividades (ZIELINSKI \& SCHIMITT, 2015).

A sala na plataforma Moodle foi criada pela pesquisadora responsável e foram incluídos artigos, videoaulas, leituras complementares com perguntas norteadoras, exemplos de exames de nasolaringoscopia e videofluoroscopia dentro da normalidade e com alterações e casos clínicos para estudo. Os artigos científicos foram localizados nas bases de dados SciELO e Lilacs, e foram incluídos artigos publicados entre 2006 e 2015 com disfagia orofaríngea como tema central. Já para a seleção das videoaulas, foram realizadas buscas na rede social YouTube com os descritores: videoaula, disfagia orofaríngea e manobras facilitadoras e protetoras. A escolha dos casos clínicos, assim como de exames da deglutição (nasofibroscopia e videofluoroscopia), foi feita a partir de diferentes etiologias de disfagias orofaríngeas referentes aos pacientes da Clínica de Fonoaudiologia da FOB/ USP que já tinham assinado o termo de autorização de dados para pesquisa.

Com isso, foi possível dividir todo o conteúdo pedagógico em seis tópicos: fisiologia da deglutição, definição e etiologia da disfagia orofaríngea, avaliação da deglutição, terapia da disfagia orofaríngea, casos clínicos e material complementar.

Dentre as ferramentas que foram utilizadas para a realização das atividades estão: os questionários (instrumento com questões de verdadeiro-falso, múltipla escolha e discursivas); diários de reflexão (ferramenta individual em que há possibilidade de construir um texto de reflexão ou resumo de aprendizagem sobre determinado tema) e tarefa (trabalho a ser realizado e enviado pelos estudantes, como relatórios, criação de imagens, textos em geral, entre outros) (MACHADO, 2012, pp. 1-16). Todas as atividades foram monitoradas pela docente e pesquisadora responsável, sendo fornecidos comentários e feedbacks. As atividades foram acessadas e respondidas de maneira individual por cada estudante, sendo que não foi permitido o acesso às respostas uns dos outros e, além disso, os estudantes receberam, no início da pesquisa, um cronograma com as datas e prazos de entrega das atividades.

\section{Avaliação de Desempenho}

Ambos os grupos foram avaliados quanto aos aspectos cognitivos, procedimentais e atitudinais. Para analisar o conhecimento cognitivo foi aplicado um questionário (APÊNDICE 1) elaborado pela pesquisadora, composto por dez perguntas 
de múltipla escolha referentes ao conteúdo abordado na disciplina, com a avaliação realizada de forma presencial e individual no início e final da disciplina em ambas as turmas, a fim de verificar o conhecimento dos estudantes e a evolução dos mesmos sobre os conteúdos presentes na disciplina e, ainda, se houve diferença no desempenho entre os grupos de ensino presencial e híbrido. Durante a aplicação do questionário não foi permitido que os estudantes trocassem informações entre si ou consultassem qualquer material didático ou anotações. Ao final da pesquisa os acertos e erros foram mostrados aos estudantes individualmente, assim como o gabarito de respostas.
A fim de avaliar os aspectos cognitivos, procedimentais e atitudinais em diferentes situações de aprendizagem, utilizou-se um Protocolo de Avaliação de Habilidades e Competências (Figura 1).

- Aspectos Cognitivos: refere-se à participação do estudante durante aulas teóricas, seminários e supervisão, considerando o domínio do caso atendido, questionamentos pertinentes durante a discussão dos casos e articulação entre teoria e prática.

- Aspectos Procedimentais: refere-se à performance durante os atendimentos aos pacientes, considerando o domínio na realização de história clínica, aplicação de protocolos específicos,

\begin{tabular}{|c|c|c|c|c|}
\hline \multicolumn{5}{|c|}{$\begin{array}{l}\text { Disciplina: Clínica de Disfagia Orofaríngea } \\
\text { Estudante: } \\
\text { Paciente: }\end{array}$} \\
\hline ASPECTOS & SITUAÇÕES & $\begin{array}{c}\text { TÓPICOS } \\
\text { AVALIADOS }\end{array}$ & -1 & - \\
\hline Cognitivos & $\begin{array}{l}\text { - Aula teórica } \\
\text { - Supervisão } \\
\text { - Seminário }\end{array}$ & $\begin{array}{l}\text { - Participação; } \\
\text { - Domínio do caso atendido; } \\
\text { - Intervenções, questiona- } \\
\text { mentos; } \\
\text { - Articulação entre teoria e } \\
\text { prática relacionada aos casos } \\
\text { clínicos. }\end{array}$ & & \\
\hline Procedimentais & $\begin{array}{l}\text { - Atendimento } \\
\text { ao paciente }\end{array}$ & $\begin{array}{l}\text { - Domínio na história clínica } \\
\text { e aplicação de protocolos } \\
\text { específicos; } \\
\text { - Domínio nos procedimen- } \\
\text { tos envolvidos na avaliação } \\
\text { clínica; } \\
\text { - Domínio na condução e } \\
\text { execução dos exercícios mio- } \\
\text { funcionais orofaciais, vocais, } \\
\text { posturais, respiratórios e } \\
\text { funcionais. }\end{array}$ & & \\
\hline Atitudinais & $\begin{array}{l}\text { - Atendimento } \\
\text { ao paciente }\end{array}$ & $\begin{array}{l}\text { - Capacidade de escuta ativa, } \\
\text { empatia e acolhimento; } \\
\text { - Preparo e organização das } \\
\text { sessões de terapia. }\end{array}$ & & \\
\hline
\end{tabular}

Figura 1 - Protocolo de avaliação de habilidades e competências. 
procedimentos envolvidos na avaliação clínica, condução e execução das estratégias terapêuticas.

- Aspectos Atitudinais: refere-se à capacidade de escuta ativa, empatia e acolhimento durante os atendimentos aos pacientes, além da responsabilidade com o preparo e organização das sessões de terapia.

O referido protocolo foi aplicado a cada estudante de acordo com as observações da docente responsável, uma vez que esta acompanhou a evolução dos estudantes durante todos os atendimentos. Para cada um dos itens do protocolo, a docente responsável fornecia uma nota de 0 a 10 para o desempenho do discente.

Além disso, os estudantes do grupo 2 responderam três perguntas sobre o conteúdo on-line apresentado, sendo que na primeira questão o estudante precisava responder o quanto o material on-line o ajudou na disciplina, podendo responder: "não ajudou" (pontuação 0); "ajudou pouco" (pontuação 1); "ajudou" (pontuação 2) e "ajudou muito" (pontuação 3). As últimas perguntas eram discursivas e solicitavam que o estudante expressasse o que acreditava estar bom no material e, por fim, o que ele gostaria que melhorasse.

\section{Análise Estatística}

Os dados foram analisados de forma quantitativa e qualitativa. A comparação entre as notas obtidas no questionário antes e depois da disciplina foi realizada por meio da aplicação do teste estatístico t de Student, a fim de saber se essa diferença era estatisticamente significante $(p<0,05)$. A média das notas entre ambos os grupos foi comparada nas avaliações de competências e habilidades. A percepção dos estudantes sobre a contribuição do ambiente virtual de aprendizagem no processo educacional foi analisada descritivamente.

\section{Resultados}

As respostas de cada estudante no questionário cognitivo foram analisadas, tanto no início quanto no final do estágio, para que fosse possível comparar o desempenho das turmas por meio da aplicação do questionário, como mostra a tabela 1.
Antes de cursar a disciplina os grupos apresentaram desempenhos heterogêneos, com melhor desempenho para o grupo $1(\mathrm{p}=0,002)$. Após a disciplina, a reaplicação do questionário demonstrou que ambos os grupos passaram a ter desempenho semelhante $(\mathrm{p}=0,120)$. A comparação entre os resultados obtidos dentro de cada grupo confirmou a efetividade do ensino a distância associado ao presencial, pois foi verificado que o ensino híbrido apresentou diferença estatisticamente significante $(\mathrm{p}<0,05)$, ao contrário do ensino presencial exclusivamente, que não apresentou evolução equivalente ao ensino híbrido.

As notas foram atribuídas pela docente responsável a partir das habilidades e competências referentes aos aspectos cognitivos, procedimentais e atitudinais construídos por cada um dos estudantes após a conclusão da disciplina "Clínica de Disfagia Orofaríngea - Estágio Supervisionado", sendo que em cada um dos aspectos a nota máxima que poderia ser obtida era 10. Os resultados estão apresentados na tabela 2 .

A aplicação do teste estatístico demonstrou que o grupo 1 manteve o melhor desempenho no aspecto cognitivo em relação ao grupo 2 , com diferença estatisticamente significante $(p=0,003)$. Por outro lado, o grupo 2 apresentou melhor desempenho no aspecto procedimental em relação ao grupo 1, também com diferença estatisticamente significante $(\mathrm{p}=0,013)$. Para o aspecto atitudinal, ambos os grupos finalizaram a disciplina com desempenho excelente, sem diferença entre as modalidades de ensino $(\mathrm{p}>0,05)$.

Quanto às questões sobre o material pedagógico on-line respondidas pelo grupo 2, 57\% afirmaram que o material ajudou muito, enquanto $43 \%$ afirmaram que o material ajudou.

Sobre o que os estudantes acreditavam que estava bom no material, os relatos foram (sic):

Com as atividades para responder, desde o início da disciplina até o final, foi possível identificar o que eu precisava estudar mais para melhorar meu desempenho. Além disso, com as atividades conseguimos ver a nossa evolução no início do 


\begin{tabular}{|c|c|c|c|}
\hline Grupos & Pré & Pós & Valor $p$ \\
\hline \multirow{8}{*}{$\begin{array}{c}\text { G1 - Ensino } \\
\text { Presencial }\end{array}$} & 8,4 & 9,0 & \multirow{8}{*}{$=0,14$} \\
\hline & 9,0 & 10,0 & \\
\hline & 8,4 & 8,8 & \\
\hline & 8,8 & 9,0 & \\
\hline & 8,8 & 7,8 & \\
\hline & 9,0 & 10,0 & \\
\hline & 6,6 & 9,8 & \\
\hline & 10,0 & 9,0 & \\
\hline $\begin{array}{c}\text { Média } \pm \text { Desvio } \\
\text { Padrão }\end{array}$ & $8,6 \pm 0,95$ & $9,1 \pm 0,74$ & \multirow{8}{*}{$=0,005^{*}$} \\
\hline \multirow{7}{*}{$\begin{array}{c}\text { G2 - Ensino } \\
\text { Híbrido }\end{array}$} & 8,0 & 8,8 & \\
\hline & 4,5 & 8,0 & \\
\hline & 6,0 & 8,6 & \\
\hline & 7,5 & 9,8 & \\
\hline & 7,0 & 8,6 & \\
\hline & 5,5 & 7,6 & \\
\hline & 9,0 & 8,6 & \\
\hline $\begin{array}{c}\text { Média } \pm \text { Desvio } \\
\text { Padrão }\end{array}$ & $6,7 \pm 1,55$ & $8,5 \pm 0,68$ & \\
\hline
\end{tabular}

Tabela 1 - Apresentação dos resultados obtidos por meio da aplicação do questionário pré e pós-disciplina (aspectos cognitivos). *Estatisticamente significante.

\begin{tabular}{|c|c|c|c|c|c|c|}
\hline \multirow[t]{10}{*}{ Aspectos } & \multicolumn{2}{|c|}{ Cognitivo } & \multicolumn{2}{|c|}{ Procedimental } & \multicolumn{2}{|c|}{ Atitudinal } \\
\hline & G1 & $\mathrm{G} 2$ & G1 & G2 & G1 & G2 \\
\hline & 9,3 & 7,2 & 10,0 & 10,0 & 10,0 & 10,0 \\
\hline & 8,2 & 7,1 & 10,0 & 10,0 & 10,0 & 10,0 \\
\hline & 7,8 & 7,2 & 8,6 & 10,0 & 10,0 & 10,0 \\
\hline & 9,0 & 6,5 & 8,6 & 10,0 & 10,0 & 10,0 \\
\hline & 7,8 & 6,5 & 8,6 & 10,0 & 10,0 & 10,0 \\
\hline & 7,1 & 6,7 & 9,3 & 10,0 & 10,0 & 10,0 \\
\hline & 7,2 & 7,0 & 10,0 & 10,0 & 10,0 & 10,0 \\
\hline & 8,8 & & 9,3 & & 10,0 & \\
\hline $\begin{array}{l}\text { Média } \pm \text { Desvio } \\
\quad \text { Padrão }\end{array}$ & $8,1 \pm 0,82$ & $6,8 \pm 0,31$ & $9,3 \pm 0,64$ & $10,0 \pm 0$ & $10,0 \pm 0$ & $10,0 \pm 0$ \\
\hline Valor $p$ & \multicolumn{2}{|c|}{$p=0,003^{*}$} & \multicolumn{2}{|c|}{$p=0,013^{*}$} & \multicolumn{2}{|c|}{$p>0,05$} \\
\hline
\end{tabular}

Tabela 2 - Apresentação do desempenho dos estudantes em cada aspecto avaliado por meio do protocolo de habilidades e competências. ${ }^{*} \mathrm{p}<0,05$ nível de significância. $\mathbf{G 1}=$ grupo $1 ; \mathbf{G} 2=$ grupo 2 . 
semestre e quando ele se encerrou, por meio das dificuldades apresentadas na hora de responder cada questão. Acredito que as disponibilizações dos vídeos no Moodle também ajudaram bastante. Então a professora usava termos em aula e depois tinha o vídeo ilustrando o que ela queria dizer, deixando tudo mais claro;

Gostei bastante dos vídeos e artigos porque era exatamente o que era abordado no estágio;

Gostei porque tinham vários vídeos e conteúdos em formas de casos, assim como os questionários;

O material estava didático, os conteúdos estavam objetivos e de fácil entendimento;

Gostei, principalmente, das videoaulas e dos casos clínicos;

O conteúdo e a maneira que ele foi apresentado. Achei muito importante ler cada caso clínico e ter um material com a linha de raciocínio completa para estudar. Além disso, os questionários mostraram os pontos que precisavam ser revisados;

Havia bastante material de estudo para auxiliar os alunos, assim como os exercícios.

Por fim, quanto ao que gostariam que melhorasse, um estudante sugeriu que existissem mais questionários na plataforma, não havendo sugestões de outros estudantes.

\section{Discussão}

O Moodle é um ambiente virtual de aprendizado que tem sido reconhecido e utilizado com bastante frequência em disciplinas de cursos de graduação (OLIVEIRA, 2017, pp. 175-178; SARAIVA, MULLER \& VEIT, 2015, pp. 3504-20; ABEGG, BASTOS \& MULLER, 2010, pp. 205-218), uma vez que seus benefícios incluem o acesso rápido à informação, a atualização rápida, o aprendizado supervisionado e a disponibilidade de ferramentas para avaliação dos estudantes (RIVA, 2000, pp. 989-98).

As diversas áreas da saúde já obtiveram sucesso no desenvolvimento de conteúdos na Plataforma Moodle, como na área de Nutrição (PATRICIO, 2010), Enfermagem (DOMENICO \& COHRS, 2016, pp. 381-389; PEREIRA et al., 2018, pp. 49-57), Medicina (MEZZARI et al., 2012, pp. 557563), entre outros. Em relação à Fonoaudiologia, a literatura contempla estudos na área de audiologia (CORRÊA et al., 2015, pp. 268-280), motricidade orofacial (CORRÊA et al., 2016) e voz (CORRÊA et al., 2012), além de um trabalho interdisciplinar com o curso de Odontologia (RAMOS et al., 2015, pp. 101-121), no qual foi usada a mesma plataforma.

Em relação à área de disfagia orofaríngea, a literatura mostra ausência de estudos de ensino a distância voltados para a graduação (CATALANI, LUCGAS \& BERRETIN-FELIX, 2016, pp. 638-648). Como outras áreas da saúde obtiveram sucesso na implementação dessa modalidade em suas disciplinas, e considerando que a área de disfagia orofaríngea é atualmente um campo de trabalho a ser explorado por fonoaudiólogos recém-formados, faz-se necessário que sejam desenvolvidos estudos investigando a eficácia de plataformas como o Moodle associadas ao ensino presencial, a fim de obter melhoras no desempenho teórico-prático dos estudantes. Diante dessa necessidade, justifica-se o desenvolvimento do presente trabalho.

A literatura contempla estudos que abordam a utilização da tele-educação em disfagia orofaríngea, tendo como público-alvo os fonoaudiólogos graduados (DAVIS \& COPELAND, 2005, pp. 141-148; SHARMA et al., 2011, pp. 688-695; GUTHRIE, LANCASTER \& STANSFIELD, 2017, pp. 854-869). Frente a isso, diferentemente nesta pesquisa foi aplicado o ensino a distância com estudantes de graduação na área de disfagia orofaríngea, assim como foram utilizadas diferentes formas de avaliação do conhecimento: o questionário, o protocolo de avaliação de 
habilidades e competências e, ainda, o questionário de percepção dos estudantes quanto ao material virtual desenvolvido.

A fim de avaliar os estudantes participantes desta pesquisa, foram considerados temas comuns aos citados na pesquisa de Guthrie, Lancaster e Stansfield (2017), como habilidades práticas, supervisão e planejamento de trabalho, essenciais para o desenvolvimento da capacitação de profissionais em disfagia orofaríngea.

Assim como nas pesquisas de Davis e Copeland (2005), Sharma et al. (2012) e Ramos et al. (2015), neste estudo se utilizou um questionário objetivo pré e pós-treinamento a distância, para mensurar o nível de conhecimento dos participantes e, também, foram obtidos resultados estatisticamente significantes apenas no grupo que passou pelo ensino a distância.

Uma vez que os grupos que passaram pelas modalidades de ensino presencial e híbrido eram heterogêneos antes da disciplina, pode-se deduzir que o ensino híbrido tenha colaborado com a melhora estatisticamente significativa do segundo grupo, porém não é possível afirmar que essa seja a única razão. Nenhum dos estudos encontrados comparou o desempenho dos ensinos presencial e híbrido, impossibilitando a comparação dos achados do presente estudo com a literatura.

Porém, para os aspectos cognitivos analisados por meio do Protocolo de Avaliação de Habilidades e Competências, o grupo presencial manteve o melhor desempenho, enquanto o contrário foi observado para os aspectos procedimentais. Uma possível explicação para a diferença em relação aos aspectos cognitivos seria o perfil de desempenho dos grupos estudados, já verificado na avaliação inicial. Em relação aos achados superiores do grupo de ensino híbrido nos aspectos procedimentais, é importante considerar que os estudantes obtiveram contato com material educacional on-line, o que possibilitou ver e rever procedimentos de avaliação e intervenção além da sala de aula. Na medida em que combina a educação presencial com a on-line, o ensino híbrido permite que o estudante tenha mais flexibilidade e autonomia para estudo, o que acarreta melhor aproveitamento do conteúdo e das atividades presenciadas em sala de aula (IGLESIAS, BERRADE \& ASTRAY, 2009, pp. 117-124).

Ressalta-se que era esperado o bom desempenho dos estudantes de ambos os grupos nos aspectos procedimentais e atitudinais, já que todos tiveram acesso a práticas clínicas e simulação, observação e execução de atendimentos a pacientes com disfagia orofaríngea durante as atividades do ensino presencial.

A aprovação do grupo híbrido sobre o material on-line desenvolvido pode ser atribuída à abrangência de temas inclusos no tópico de disfagia orofaríngea e às diversas formas de apresentação e avaliação dos conteúdos, já que esse complementava e abordava temas apresentados em aulas presenciais, possibilitando revisão daqueles conteúdos e crescimento de conhecimento sobre esses. Este tópico também foi empregado no estudo de Corrêa et al. (2012), em que foi considerada a opinião dos estudantes sobre o material on-line desenvolvido e houve a aprovação quanto à qualidade dos questionários, vídeos e facilidade de navegação.

Um ponto que deve ser abordado em pesquisas futuras é a organização de grupos homogêneos de acordo com características específicas, como o desempenho acadêmico dos participantes, a fim de estabelecer maior controle e confiabilidade nos dados obtidos para a comparação dos diferentes tipos de ensino.

Por fim, deve ser considerada a relevância do presente estudo por demonstrar a importância do ensino híbrido na formação de profissionais em Fonoaudiologia, uma vez que a união entre a educação a distância e a presencial foi capaz de potencializar o desempenho dos estudantes nos aspectos cognitivos, procedimentais e atitudinais, demonstrando a necessidade da utilização de ambas as modalidades de ensino. Sugere-se ainda a continuidade de estudos, ampliando a metodologia proposta na presente pesquisa em outros grupos de estudantes e para outras disciplinas do curso de graduação em Fonoaudiologia, visto que esse é um tema pouco abordado. 


\section{Conclusão}

O ensino a distância associado ao presencial foi efetivo para o processo de aprendizagem dos estudantes de graduação de Fonoaudiologia na área de Disfagia Orofaríngea, uma vez que contou com a aprovação dos estudantes que utilizaram o ensino híbrido e, além disso, foi responsável por maior evolução no desempenho desses estudantes quando comparado ao ensino exclusivamente presencial.

\section{Referências Bibliográficas}

ABEGG, Ilse; BASTOS, F. P. \& MULLER, F. M. "Ensino-Aprendizagem Colaborativo Mediado pelo Wiki do Moodle". Educ. Rev., n. 38, 2010, pp. 205-218. Disponível em: <http:// www.scielo.br/scielo.php?script=sci_arttext\&pi$\mathrm{d}=\mathrm{S} 0104-40602010000300014 \& \operatorname{lng}=\mathrm{en} \& \mathrm{nrm}=\mathrm{iso}>$. Acessado em 03 abr. 2019.

ABDULMASSIH, Edna Márcia da Silva; MACEDO FILHO, E. D.; SANTOS, R. S. \& JURKIEWICZ, A. L. "Evolução de Pacientes com Disfagia Orofaríngea em Ambiente Hospitalar". Arquivo Internacional de Otorrinolaringologia, São Paulo, vol. 13, n. 1, 2009, pp. 55-62.

BAIJENS, Laura W.; KOETSENRUIJTER, K. \& PILZ, W. "Diagnosis and Treatment of Phagophobia: a Review". Dysphagia, vol. 28, n. 2, 2013, pp. 260-70.

CASTRO CORRÊA, Camila; MARTINS, A.; PARDO-FANTON, G.; DA SILVA, A.S.C.; BARROS, G.T.T.;WEN, C.L.;MAXIMINO,L.P.;BERRETINFELIX, G.; BLASCA, W. Q. \& BRASOLOTTO, A. G. "Ações de Teleducação Interativa em Saúde Vocal Baseadas na Dinâmica do Projeto Jovem Doutor". Distúrbios da Comunicasãa, vol. 24, n. 3, 2012, pp. 359-368.

CASTRO CORRÊA, Camila; SILVA, R. A.; BARROS, G. T.; PARDO-FANTON, C. \& BLASCA, W. Q. "Promoção da Saúde Auditiva Baseada na Dinâmica do Projeto Jovem Doutor". Distúrbios da Comunicaşão, vol. 27, n. 2, 2015, pp. 268-280.

CASTRO GORRÊA, Camila; BERRETINFELIX, G. \& BLASCA, W. Q. "Educational Program Applied to Obstructive Sleep Apnea". Journal of Communication Disorders, Deaf Studies \& Hearing Aids, vol. 4, n. 2, 2016.

CATALANI, Brenda; DE LUCCAS, G. R.; BERRETIN-FELIX, G. "Tele-Educação e Teleatendimento em Disfagia Orofaríngea: Revisão de Literatura". Distúrbios da Comunicaşão, vol. 28, n. 4, 2016, pp. 638-648.

CLAVÉ, Pere; TERRÉ, R.; DE KRAA, M. \& SERRA, M. "Approaching Oropharyngeal
Dysphagia". Rev Esp Enferm Dig, Madrid, vol. 96, n. 2, 2004, pp. 119-131.

CÓDIGO de Ética da Fonoaudiologia. Resolução 138/95 do Conselho Federal de Fonoaudiologia. Disponível em: <https://www.fonoaudiologia.org. $\mathrm{br} / \mathrm{cffa} /$ index.php/codigo-de-etica/ $>$. Acessado em 18 abr. 2019.

CRARY, Michael A.; CARNABY, G. D.; GROHER, M. E. \& HELSETH, E. "Functional Benefits of Dysphagia Therapy Using Adjunctive sEMG Biofeedback". Dysphagia, vol. 19, 2004, pp. 160-164.

DAVIS, Lori \& COPELAND, K. "Effectiveness of Computer-Based Dysphagia Training for Direct Patient Care Staff". Dysphagia, vol. 20, n. 2, 2005, pp. 141-148.

DOMENICO, Edvane Birelo Lopes de \& COHRS, C. R. "Plataforma Moodle na Construção do conhecimento em Terapia Intensiva: Estudo Experimental". Acta Paul. Enferm., vol. 29, n. 4, 2016, pp. 381-389.

GONÇALVES, Maria Inês Rebelo; REMAILI, C. B. \& BEHLAU, M. "Equivalência Cultural da Versão Brasileira do Eating Assessment Tool - EAT - 10". CoDAS, São Paulo, vol. 25, n. 6, 2013, pp. 601-604.

GOVENDER, Roganie; SMITH, C. H.; TAYLOR, S. A.; BARRATT, H. \& GARDNER, B. "Swallowing Interventions for the Treatment of Dysphagia after Head and Neck Cancer: a Systematic Review of Behavioural Strategies Used to Promote Patient Adherence to Swallowing Exercises". BMC Cancer, vol. 17, n. 1, 2017, p. 43.

GUTHRIE, Susan; LANCASTER, J. \& STANSFIELD, J. "Consensus-Building on Developing Dysphagia Competence: a North West of England Perspective". International Journal of Language \& Communication Disorders, vol. 52, n. 6, 2017, pp. 854-869.

IGLESIAS, Beatriz Gal; BERRADE, B. I. \& ASTRAY, G. M. C. "Nuevas Metodologias Docentes Aplicadas al Estúdio de la Fisiologia y la Anatomia: Estúdio Comparativo com el Método Tradicional". Rev Bras Educ Med., vol. 12, n. 2, 2009, pp. 117-124.

LANGMORE, Susan E.; MCGULLOCH, T. M.; KRISGIUNAS, G. P.; LAZARUS, C.L.; VAN DAELE, D. J.; PAULOSKI, B. R.; RYBIN, D. \& DOROS, G. "Efficacy of Electrical Stimulation and Exercise for Dysphagia in Patients with Head and Neck Cancer: A Randomized Clinical Trial". Head Neck, vol. 38, n. S1, 2016, pp. E1221-31.

MACHADO, Claudia. "As Ferramentas de Comunicação do Moodle como Apoio a uma Unidade Curricular de um Curso de Licenciatura". Rev Educa Online, Rio de Janeiro, vol. 6, n. 2, 2012.

MACHADO, Renata Santos Pereira; COELHO, M. A. S. C. \& VERAS, R. P. "Validity of the Portuguese 
Version of the Mini Nutritional Assessment in Brazilian Elderly". BMC Geriatrics, vol. 15, 2015, pp. 132.

MEZZARI, Adelina; ISER, I.; WIEBBELLING, A. M. P. \& TAROUCO, L. "O Uso do Moodle como Reforço ao Ensino Presencial de Parasitologia e Micologia no Gurso de Graduação em Medicina". Rev. Bras. Educ. Med., vol. 36, n. 4, 2012, pp. 557-563.

MILES, Anna \& ALLEN, J. "Management of Orop haryngeal Neurogenic Dysphagia in Adults". Curr Opin Otolaryngol Head Neck Surg., vol. 23, n. 6, 2015, pp. 433-9.

OLIVEIRA, Elisabeth Andreoli. "Uso de Questionários no Moodle como Apoio para Aprendizagem". Revista de Graduação USP, vol. 2, n. 3, 2017, pp. 175-178.

PADOVANI, Aline Rodrigues; MORAES, D. P.; SASSI, F. C. \& ANDRADE, C. R. F. de. "Avaliação Clínica da Deglutição em Unidade de Terapia Intensiva". CoDAS, São Paulo, vol. 25, n. 1, 2013, pp. 1-7.

PADOVANI, Aline Rodrigues; MORAES, D. P.; MANGILI, L. D. \& ANDRADE, G. R. F. de. "Protocolo Fonoaudiológico de Avaliação do Risco para Disfagia (Pard)". Rev Soc Bras Fonoaudiol., vol. 12, n. 3, 2007, pp. 199-205.

PARK, Jun Seong; OH, D. H.; HWANG. N. K. \& LEE, J. H. "Effects of Neuromuscular Electrical Stimulation Combined with Effortful Swallowing on Post-Stroke Oropharyngeal Dysphagia: a Randomised Controlled Trial". J Oral Rehabil. vol. 43, n. 6, 2016, pp. 426-34.

PATRICIO, Roberta Saraiva Giroto. Desenvolvimento de Material Educativo Digital para Divulgação Científica da Nutrigenômica. Dissertação (Mestrado em Ciência dos Alimentos), Faculdade de Ciências Farmacêuticas, Universidade de São Paulo, São Paulo, 2010.

PEREIRA, Marta Cristiane Alves; ISAAC, I.; BODNAR, J.; MORAES, J.; SILVA, G. \& CAMARGO, R. "Avaliação da Aprendizagem em Gerenciamento de Custos de Materiais Aplicado à Enfermagem Hospitalar". Revista de Graduação USP, vol. 3, n. 1, 2018, pp. 49-57.

PERRY, Alison; LEE, S. H.; COTTON, S. \& KENNEDY, C. "Therapeutic Exercises for Affecting Post-Treatment Swallowing in People Treated for Advanced-Stage Head and Neck Cancers". Cochrane Database Syst Rev, n. 8, 2016.

PICCOLI, Gabriele; AHMAD, Rami \& IVES, Blake. "Web-Based Virtual Learning Environments: A Research Framework and a Preliminary Assessment of Effectiveness in Basic IT Skills Training". MISQuarterly, vol. 25, n. 4, dez. 2001, pp. 401-426.

PORTAS, Juliana \& GUEDES, R. L. V. "Protocolo de Qualidade de Vida em Deglutição". In: CARVALHO, V. \& BARBOSA, E. A. Fononcologia. Rio de Janeiro: Revinter, cap.10, 2012, pp. 169-92.
RAMOS, Janine Santos; DA SILVA, L. K.; PINZAN, A.; RODRIGUES, A. C. \& BERRETINFELIX, G. "Distance Learning: Effectiveness of an Interdisciplinary Course in Speech Pathology and Dentistry". Interdisciplinary Journal of e-Skills and Lifelong Learning, vol. 11, 2015, pp. 101-121.

RIVA, Giuseppe. "From Telehealth to e-Health: Internet and Distributed Virtual Reality in Health Care". Cyber Psych Behav, vol. 3, n. 6, 2000, pp. 989-98.

SANTORO, Patricia; E SILVA, I. L.; CARDOSO, F.; DIAS JR., E. \& BERESFORD, H. "Evaluation of the Effectiveness of a Phonoaudiology Program for the Rehabilitation of Dysphagia in the Elderly". Arch Gerontol Geriatr., vol. 53, n. 1, 2011, pp. 61-6.

SHARMA, Shobha; WARD, E. C.; BURNS, C.; THEODOROS, D. \& RUSSELL, T. "Assessing Swallowing Disorders Online: a Pilot Telerehabilitation Study". Telemedicine and e-Health, vol. 17, n. 9, 2011, pp. 688-695.

SARAIVA, Maria de Fátima Oliveira; MULLER, A. M. \& VEIT, E. A. "Fundamentos de Astronomia e Astrofísica na Modalidade a Distância: Uma Disciplina para Alunos de Graduação em Física". Rev. Bras. Ensino Fís., vol. 37, n. 3, 2015, pp. 3504-20.

SHARMA, Shobha; WARD, E. C.; BURNS, C.; THEODOROS, D. \& RUSSELL, T. "Training the Allied Health Assistant for the Telerehabilitation Assessment of Dysphagia". Journal of Telemedicine and Telecare, vol. 18, n. 5, 2012, pp. 287-291.

SPEYER, Reneé; BAIJENS, L.; HEIJNEN, M. \& ZWIJNENBERG, I. "Effects of Therapy in Oropharyngeal Dysphagia by Speech and Language Therapists: a Systematic Review". Dysphagia, vol. 25, n.1, 2010, pp. 40-65.

VALE-PRODOMO, Luciana Passuello; ANGELIS, E. C. \& BARROS, A. P. B. "Avaliação Clínica Fonoaudiológica das Disfagias". In: JOTZ, G. P.; ANGELIS, E. C. \& BARROS, A. P. B. Tratado da Deglutição e Disfagia: No Adulto e na Criança. Rio de Janeiro: Revinter, cap. 6, 2010, pp. 61-67.

WIRTH, Rainer, DZIEWAS, R.; BEGK, A.M.; CLAVÉ, P.; HAMDY, S.; HEPPNER, H.J.; LANGMORE, S.; LEISCHKER, A.H.; MARTINO, R.; PLUSCHINSKI, P.; RÖSLER, A.; SHAKER, R.; WARNEGKE, T.; SIEBER, C. C. \& VOLKERT, D. "Oropharyngeal Dysphagia in Older Persons - from Pathophysiology to Adequate Intervention: a Review and Summary of an International Expert Meeting". Clin Interv Aging, vol. 11, 2016, pp. 189-208. Disponível em: $<$ https://www.ncbi.nlm.nih.gov/pubmed/26966356>. Acessado em 28 abr. 2019.

WEN, Chao Lung. "Telemedicina e Telessaúde: um Panorama no Brasil". Informática Pública, vol. 10, n. 2, 2008, pp. 7-15.

ZIELINSKI, Felipe Dias Costa \& SCHMITT, M. A. R. "Uma ferramenta gráfica para suporte à atividade docente no Moodle". Renote, vol. 13, n. 1, 2015.

Publicado em 28/07/2020. 


\section{APÊNDICE 1 - Questionário}

\section{QUESTIONÁRIO DA PESQUISA: "Educação Mediada por Tecnologia em Disfagia Orofaríngea: Proposta de Ensino na Graduação"}

Identificação do participante

Nome:

Idade: Data:

( ) Pré disciplina

( ) Pós disciplina

Responda as questões abaixo de acordo com o enunciado. Ao finalizar, favor entregar este questionário à pesquisadora.

Questão 1: O que é disfagia?

a) É uma alteração nutricional relacionada a uma doença de base.

b) É uma dificuldade de mastigar que causa alteração na fase oral da deglutição, sem prejuízo nas demais fases.

c) É uma alteração da deglutição que ocorre apenas em doenças neurológicas.

d) É uma doença que irá causar nos pacientes uma complicação hospitalar chamada pneumonia aspirativa.

e) É uma dificuldade de deglutir ou a dificuldade na transferência do bolo alimentar da boca ao estômago, que pode ocorrer nas diferentes fases da deglutição.

Questão 2: As disfagias mecânicas ocorrem em indivíduos:

a) Com doenças do sistema digestivo.

b) Com problemas respiratórios.

c) Com distúrbios psicológicos.

d) Com câncer de cabeça e pescoço.

e) Que sofreram perda de peso.

Questão 3: Assinale V para as alternativas verdadeiras e F para as falsas.

() A disfagia pode ser congênita ou adquirida.

( ) O tratamento da disfagia orofaríngea e esofágica é de competência do fonoaudiólogo.

( ) A avaliação clínica da disfagia é feita após a realização do exame instrumental.

( ) Todo paciente com disfagia terá episódios de penetração e aspiração.

( ) A videofluroscopia é considerada o exame instrumental padrão ouro para avaliar a função da deglutição.

Questão 4: O que é penetração?

a) Ocorre quando o alimento é deglutido e entra no vestíbulo laríngeo, mas não passa o nível das pregas vocais.

b) Ocorre quando o alimento fica parado no esôfago.

c) Ocorre quando o alimento é deglutido e segue para o estômago.

d) Ocorre quando o alimento é deglutido, passa as pregas vocais e segue para a via aérea.

e) Ocorre quando o alimento é deglutido e observam-se resíduos na valécula.

Questão 5: O que é aspiração?

a) Ocorre quando o paciente tem nasorregurgitação.

b) Ocorre quando o alimento é deglutido e observam-se resíduos na valécula.

c) Ocorre quando o alimento é deglutido, passa as pregas vocais e segue para a via aérea.

d) Ocorre quando o alimento fica parado no esôfago.

e) Ocorre quando o alimento é deglutido e entra no vestíbulo laríngeo, mas não passa o nível das pregas vocais. 
Questão 6: Como é feito o tratamento fonoaudiológico das disfagias?

a) Apenas com exercícios miofuncionais, vocais e respiratórios.

b) Com medicação, modificação de volumes e consistências e uso de espessantes.

c) Com exercícios mastigatórios e fonoarticulatórios.

d) Com via alternativa de alimentação, modificação da postura corporal e estimulação manual.

e) Com exercícios miofuncionais, vocais e respiratórios; manobras protetoras e facilitadoras; modificação de volumes e consistências.

Questão 7: Sobre a modificação de volumes e consistências e via alternativa de alimentação, assinale $\mathrm{V}$ para as alternativas verdadeiras e $\mathrm{F}$ para as falsas.

( ) Todo paciente diagnosticado com disfagia deve utilizar espessantes alimentares no início do tratamento.

( ) Apenas pacientes com disfagia de grau moderado e severo precisam realizar mudanças de volumes e consistências.

( ) A modificação de consistências é orientada pelo médico e seguida pelo fonoaudiólogo.

( ) A indicação de via alternativa de alimentação é realizada em conjunto com fonoaudiólogo, médico e nutricionista.

( ) Pacientes com via alternativa de alimentação não podem comer nada por via oral.

Questão 8: Em relação à finalidade dos exercícios vocais para pacientes com disfagia, qual alternativa incorreta?

a) Para melhorar a elevação laríngea e melhorar coaptação glótica.

b) Exercícios vocais são indicados apenas para pacientes que também possuam disfonia, além do quadro de disfagia.

c) Para melhorar a elevação laríngea, coaptação glótica e controle do fluxo aéreo.

d) Para melhorar a elevação laríngea e a coaptação glótica.

e) Para melhorar a elevação laríngea, coaptação glótica, controle do fluxo aéreo e aumentar a proteção das vias aéreas.

Questão 9: Com qual finalidade são utilizadas as manobras de queixo para baixo, deglutição com esforço, Mendelson e Masako, respectivamente?

a) Proteger via aérea inferior; otimizar a passagem do bolo pela orofaringe; aumentar a elevação laríngea e a abertura do esfíncter esofágico superior; aumentar a movimentação da parede posterior da faringe durante a deglutição.

b) Facilitar a ejeção do bolo para a faringe; otimizar a passagem do bolo pela orofaringe; aumentar a elevação laríngea e a abertura do esfíncter esofágico superior; aumentar a movimentação da parede posterior da faringe durante a deglutição.

c) Proteger via aérea inferior; otimizar a passagem do bolo pela orofaringe; aumentar a elevação laríngea e a abertura do esfíncter esofágico superior; coordenar respiração e deglutição.

d) Proteger via aérea inferior; otimizar a passagem do bolo pela orofaringe; melhorar a função de lábios e língua; aumentar a movimentação da parede posterior da faringe durante a deglutição.

e) Proteger via aérea inferior; desencadear o reflexo de tosse caso seja necessário; aumentar a elevação laríngea e a abertura do esfíncter esofágico superior; aumentar a movimentação da parede posterior da faringe durante a deglutição.

Questão 10: Quais alterações podemos encontrar durante o exame de videofluoroscopia da deglutição que não podem ser visualizadas ao exame de nasoendoscopia da deglutição?

a) Penetração, aspiração e resíduos em seios piriformes.

b) Escape oral posterior, resíduos em valéculas e seios piriformes.

c) Penetração, escape oral posterior e resíduos em faringe.

d) Alteração de função de lábios e língua; penetração e aspiração durante a deglutição, alteração de motilidade esofágica.

e) Atraso para início da fase faríngea, escape oral posterior e resíduos em valécula. 